\title{
Dominance and noncommutativity effects in concept conjunctions: Extensional or intensional basis?
}

\author{
GERT STORMS, PAUL DE BOECK, IVEN VAN MECHELEN, and DIRK GEERAERTS \\ Katholieke Universiteit, Leuven, Belgium
}

\begin{abstract}
Dominance and noncommutativity effects are investigated in relative clause descriptions of five conjunctive concepts (birds and pets, sports and games, vehicles and machines, office equipment and writing implements, and shoes and sports equipment). Both asymmetry phenomena are studied at the extensional level (using membership ratings) and at the intensional level (using feature-importance ratings). A clear dominance effect was found for both the membership ratings and the feature-importance ratings, whereas the noncommutativity effect emerged only occasionally in the membership ratings and almost never in the feature-importance ratings. The data suggested that the dominance effect and the much weaker noncommutativity effect have an extensional basis.
\end{abstract}

Combinations of semantic concepts have recently been the subject of many empirical studies. The main issue is what happens to concepts that are conjunctively combined into a complex concept. Different types of conceptual combinations have been investigated: adjective-noun combinations such as brown apple (Medin \& Shoben, 1988; Murphy, 1988; Smith \& Osherson, 1984; Smith, Osherson, Rips, \& Keane, 1988; Springer \& Murphy, 1992), noun-noun combinations such as house bird or engine repair (E. V. Clark, Gelman, \& Lane, 1985; H. H. Clark, 1983; H. H. Clark \& Marshall, 1981; Downing, 1977; Kunda, Miller, \& Claire, 1990; Wisniewski \& Gentner, 1991), and relative clause descriptions of the type Xs that are also Ys (Chater, Lyon, \& Myers, 1990; Hampton, 1987) such as sports that are also games.

In these different types of conceptual combinations, the constituent concepts are modified in different ways. First, in adjective-noun combinations, noun concepts are primarily modified in the feature that is referred to in the adjective. Second, in noun-noun combinations, a noun is modified by another noun, but the modifying link can take very different forms (see, e.g., Shoben, 1991): it can be a "location" link (e.g., city bike), a "has a" link (e.g., street name), a "function" link (e.g., police car), and so on. The combinations studied in the research reported here are relative clause descriptions of concept conjunc-

This research project was supported by Grant 8.00 .84 .90 from the Belgian National Science Foundation (Fundamental Human Sciences) to P. De Boeck, I. Van Mechelen, and D. Geeraerts. The authors thank James Hampton, M. Intons-Peterson, and three anonymous reviewers for their helpful comments on an earlier version of this manuscript. We are also indebted to Ignace Hanoulle for his help in collecting the data and for his comments. Correspondence should be addressed to G. Storms, Department of Psychology, University of Leuven, Tiensestraat 102, B-3000 Leuven, Belgium (e-mail: fpaae09@blekul11). tions. The link of such relative clause descriptions is of the "is a" sort. The same link can sometimes be expressed in a noun-noun combination (e.g., pet bird), but more often this is not possible (e.g., as in the conceptual combination of weapon and tool).

In studying relative clause descriptions of concept conjunctions, two interesting asymmetry phenomena have been discovered (Chater et al., 1990; Hampton, 1987, 1988). First, in a relative clause, such as $X s$ that are also $Y s$, one of the two constituents $(X$ or $Y)$ usually has a greater influence on the conjunction than the other. This so-called "dominance" effect was identified by Hampton (1988). In predicting membership ratings for the conjunction based on the membership ratings for the two constituents, he found a clear asymmetry in the $\beta$ weights of the multiple-regression equation. The weights for one constituent concept (sports, dwellings, and birds) were significantly greater than the weights for the other constituent concept in the conjunction (games, building, and pets, respectively). The four other conjunctions used by Hampton (1988) also showed this asymmetry, but the difference between the $\beta$ weights was not significant. A similar dominance effect was found by Hampton (1987) in intensional data (i.e., feature-importance ratings). In a multiple regression predicting the importance of the features for the conjunction based on the feature ratings for the constituent concepts, the same dominance effect was found as for extensional data (i.e., membership ratings). In all but one regression equation, the dominant concept was the same as for the membership ratings. Hampton (1987) has explained this dominance effect on an intensional basis. He argues that one of the constituents in a conjunction dominates the other because the conjunction inherits more features, as well as more important features, from the dominant constituent than from the nondominant constituent. Note that, in line with a long philosophical tradi- 
tion, the notion of a concept's intension is concerned with the attributional information associated with the concept, which is used for classification or which may be inferred from that classification, whereas the notion of a concept's extension concerns the objects or entities to which the concept refers (see, e.g., Hampton, 1988; Hampton \& Dubois, 1993). In accordance with this definition, in the remainder of this paper feature-importance ratings will be referred to as intensional data and object membership ratings will be referred to as extensional data.

A second asymmetry resulted from the syntactic function of the concepts in the relative clause description: The influence of a constituent concept on the conjunction is greater when the concept is in the relative clause than when it is the head noun. In other words, the $X$ constituent is more important in $Y s$ that are also $X s$ than in $X s$ that are also Ys. For this effect, the name noncommutativity has been proposed (Hampton, 1988). Hampton found this noncommutativity effect in extensional data (membership ratings). In regression equations predicting membership of the conjunction based on the membership ratings of the constituents for a set of exemplars, Hampton found a pattern of $\beta$ weights compatible with this noncommutativity effect-that is, the $\beta$ weight for constituent $X$ is larger in the conjunction $Y_{s}$ that are also $X s$ than in the conjunction Xs that are also Ys in 10 out of 12 concept pairs. Only tools and food, in their conjunction with weapons and plants, respectively, failed to show this effect. Chater et al. (1990) also reported an overall noncommutativity effect for membership ratings, where the influence of a concept was again greater when it was in the modifying relative clause than when it was the head noun. For intensional data, the same pattern was found for defining features in 9 out of 12 concepts, with the other three showing the opposite effect (Hampton, 1987). In an overall significance test of the $\beta$ weights, when the data were aggregated over the different concepts, no significant difference was found. Hampton (1988) explained the intensional noncommutativity effect by suggesting that the conjunction inherits more features from the constituent that functions as the relative clause modifier than from the head noun constituent.

The finding of intensional dominance and noncommutativity effects is a necessary, but not sufficient, condition for an intensional basis of both asymmetry effects: The causal direction could also be the reverse, in that the effects may show up intensionally (in the feature-importance ratings) due to the mediating influence of extensional information (membership information) in the featureimportance ratings. In other words, it is possible that subjects, when asked to rate the importance of a set of features for defining a category, first think of instances of the category and then rate the importance of the features according to the applicability of the features to the generated instances.

The two asymmetry effects - the dominance and noncommutativity effects-were studied together in one design by Hampton (1988). The present research investigates the dominance and the noncommutativity effects together, but, unlike in Hampton's study, simultaneously includes in the analyses extensional and intensional variablesthat is, membership and feature-importance ratings. This enables us to control statistically for possible extensional information present in the feature-importance ratings and to control for possible intensional information present in the category membership ratings. In this way, the intensional and extensional sides of both asymmetry effects can be compared and statistical methods can be used to correct for the possibly mediating role of intensional aspects when an extensional measure is used, and vice versa. Thus, Hampton's hypothesis of an intensional basis of the dominance and noncommutativity effects can be verified in a more precise way: If the two asymmetry effects have an intensional basis (i.e., a feature-related basis), one may reasonably assume that both effects will remain in featureimportance ratings after controlling statistically for possible extensional information, whereas the two effects should disappear or be weakened in the membership ratings after controlling statistically for possible intensional information. The intensional and extensional bases of the asymmetry effects have important implications for the abstraction level at which cognitive processes take place in conceptual combination.

\section{EXPERIMENT 1}

Experiment 1 was intended to replicate Hampton's $(1987,1988)$ findings about the occurrence of dominance and noncommutativity effects in concept conjunctions and to test Hampton's hypothesis about the intensional basis of the effects. Three concept conjunctions from Hampton's (1988) study were tested.

\section{Method}

Subjects. The subjects were 120 first-year students and 30 research assistants at the Psychology Department of Leuven University. All subjects were volunteers. For the students, participation partially fulfilled a requirement of their introductory course. Each subject participated in (1) a dominance judgment task, (2) two rating tasks, one on category membership and another on feature importance, or (3) an exemplar $\times$ feature judgment task.

Material. Three conjunctive concepts, previously used by Hampton (1988), were selected. The first one was the conjunction of the semantic categories birds and pets, the second the conjunction of machines and vehicles, and the third that of sports and games. To ascertain which constituent in each pair was dominant, a second group of 80 subjects, 50 students, and 30 research assistants, completed a resemblance rating task. The subjects received two questions about each of the three conjunctions under study. They indicated how much both constituents resemble the conjunction on a 10-point scale, ranging from 1 (not at all) to 10 (very much). They also rated the resemblance of the constituents to the conjunction for only one ordering of the constituents in the conjunction. For example, the subjects were asked to indicate either how much birds and pets resemble birds that are also pets, or how much birds and pets resemble pets that are also birds. The order of the constituents in the description of the conjunction was randomized over the subjects.

Membership and feature-importance ratings. A group of 40 subjects (all of them students) participated in two rating tasks. The order of the two rating tasks was counterbalanced. One task was to rate category memberships for three lists of $\mathbf{4 0}$ exemplars: $\mathbf{4 0}$ 
animals, 40 activities, and 40 artifacts. In each list of 40 exemplars related to a conjunctive concept, each of four possible types of categories were represented with 10 exemplars. The four types of categories were the two constituent concepts (e.g., pet and bird), the conjunction (formulated in two ways-e.g., pets that are also birds and birds that are also pets), and a rest category (e.g., animals that are neither pets nor birds, artifacts that are neither machines nor vehicles, and leisure activities that are neither sports nor games). For each type of category, the 10 exemplars were selected on the basis of production frequency of these words in an exemplar-generating study (Storms, Van Mechelen, \& De Boeck, 1992). For each list of 40 exemplars, 10 subjects were asked to rate the membership of all the exemplars for four category names, with the category name representing the four category types (two constituents and the conjunction described in two ways-e.g., birds, pets, birds that are also pets, and pets that are also birds). A 7 point rating scale was used for these ratings, ranging from -3 (very unrelated words) to +3 (very typical members). Appendix $A$ lists the 40 exemplars for the sports/games conjunction.

For the other rating task, the subjects were given three lists of 30 features each. These features were selected from responses of a feature-generation study (Storms et al., 1992). The first list contained 10 features of pets, 10 features of birds, and 10 features of the conjunction of pets and birds. Similarly, the second and the third list contained 10 features of each of the two other conjunctions and 10 features of each of its constituents. ${ }^{1}$ For each list of 30 features, 10 subjects were asked to rate the importance of the features for defining four category names, representing again the four category types (two constituents and the conjunction described in two wayse.g., birds, pets, birds that are also pets, and pets that are also birds). Again, a 7-point rating scale was used, ranging from -3 (clearly unrelated features) to +3 (very important features). Appendix B lists the 30 features for the sports/games conjunction.

Exemplar $\times$ feature judgments. A group of 30 students judged the applicability of features to a set of exemplars. Ten subjects judged the 40 exemplars from the birds/pets list on the 30 corresponding features. Judgments had to be dichotomous: 1 when a feature applied, and 0 otherwise. The judgments from the 10 subjects were then added for each cell of the $40 \times 30$ matrix, resulting in an exemplar $\times$ feature applicability matrix, with entries ranging from 0 to 10 . In a similar way, exemplar $x$ feature applicability matrices were constructed for the sports and games data and for the vehicles and machines data.

\section{Results}

First, the data from the resemblance rating task were analysed. These results indicated which of the constituents in a conjunctive pair was dominant. Second, reliability measures of the membership and feature-importance ratings were obtained. Third, the dominance and noncommutativity effects were investigated in the membership ratings and in the feature-importance ratings, using analyses of variance (ANOVAs). Fourth, analyses of covariance (ANCOVAs) were computed to statistically control the possible mediating role of intensional aspects in the extensional ratings, and vice versa.

Identification of the dominant constituents. The mean resemblance ratings of both constituent concepts and their corresponding conjunction (see Table 1) show that birds resemble the pet/bird conjunction more than pets and that vehicles resemble the vehicles/machines conjunction better than machines. Sports and games show an almost equal resemblance with their conjunction. The resemblance ratings were compared in an ANOVA, with the constituent
Table 1

Mean Resemblance Ratings for Constituents and Conjunctions

$\begin{array}{ll}\text { birds } & 8.388 \\ \text { pets } & 5.663 \\ \text { sports } & 7.525 \\ \text { games } & 7.238 \\ \text { vehicles } & 7.600 \\ \text { machines } & 6.625\end{array}$

as a within-subjects factor. The mean resemblance of birds was significantly higher than that of pets $[F(1,79)=$ $52.85, p<.0001]$, and the difference between the resemblance ratings for vehicles and machines was also significant $[F(1,79)=4.87, p<.03]$. The difference between sports and games did not reach significance, but was positive, as expected. In general, these results are consistent with the findings of Hampton (1988), who reported the dominance of birds, vehicles, and sports over pets, machines, and games, respectively. The convergence of our results and the results of Hampton led us to identify these three constituents as the dominant constituents in the next analyses.

Reliability. The reliability of the membership and feature-importance ratings was estimated by applying the Spearman-Brown formula to the split-half correlation, after randomly dividing each of the subject groups who rated a set of exemplars (or a set of features) for their membership (or feature importance) in relation to the same category name into two groups of equal size. The reliability coefficients for the membership ratings in the constituents and in the conjunctions are given in Table 2 . All of these coefficients are well above .90 . The reliabilities for the corresponding feature-importance ratings are also given in Table 2. While most of these values are only slightly lower than the values for the membership ratings, clearly lower reliabilities were found for the conjunction of machines and vehicles.

The reliability of the exemplar $\times$ feature applicability judgments was estimated in a similar way as for the membership and feature-importance ratings: The SpearmanBrown formula was applied to the split-half correlation after randomly dividing the 10 subjects who made the ap-

Table 2

Estimated Reliability Coefficients of the Ratings

\begin{tabular}{lcc}
\hline & \multicolumn{2}{c}{ Ratings } \\
\cline { 2 - 3 } & Membership & Feature Importance \\
\hline birds & .997 & .964 \\
pets & .973 & .911 \\
birds that are also pets & .978 & .957 \\
pets that are also birds & .988 & .918 \\
sports & .985 & .883 \\
games & .982 & .937 \\
sports that are also games & .923 & .902 \\
games that are also sports & .984 & .937 \\
vehicles & .985 & .953 \\
machines & .988 & .873 \\
vehicles that are also machines & .925 & .650 \\
machines that are also vehicles & .994 & .698 \\
\hline
\end{tabular}


plicability judgments into two groups of 5 . The reliabilities for the birds/pets data, the sports/games data, and the vehicles/machines data were all high: .94, .91, and .95, respectively. ${ }^{2}$

Analysis of variance of the dominance and noncommutativity effects. Both asymmetry effects were investigated using correlation methods and ANOVAs. First, the correlations will be presented. Next, ANOVAs will be given, which allow us to test the statistical significance of the dominance and the noncommutativity effects, as well as their interaction.

The correlations between the membership ratings (summed over the 10 subjects who made every rating) for the constituents and the two different relative clause descriptions of the conjunction appear in Table 3. For the dominance effect, the correlations for the dominant constituents (birds, sports, and vehicles) were always higher than the corresponding correlations for the nondominant constituents. The correlations for the feature-importance ratings also appear in Table 3. Again, the dominance effect is present in both descriptions of the three conjunctions. For the noncommutativity effect, the correlations of the conjunction with a constituent were always higher when the constituent was in the relative clause rather than in the head noun, except for pets, where the reverse pattern was observed. For the feature-importance ratings, the noncommutativity effect was found for only three of the six constituents (birds, pets, and vehicles).

To test the statistical significance of the dominance and noncommutativity effects, as well as their interaction, measures of proximity between the constituents and their respective conjunctions were subjected to ANOVAs. To obtain these proximity measures, we summed the membership ratings of the 10 subjects who rated the same category type. There were four category types: the two constituents and the two descriptions of the conjunction. This resulted in four summed membership ratings (ranging from -30 to +30 ) for each exemplar and in four summed importance ratings (also ranging from -30 to +30 ) for each feature from the three feature lists. Next, these summed scores were $z$-transformed over exemplars and over features. Finally, for each exemplar and each feature, four absolute $z$-differences were derived: the difference between the ( $z$-transformed) ratings for each of the con- stituents and for the two descriptions of the conjunction. Notice that the absolute differences were always positive and that a small difference score was obtained when, for an exemplar or feature, there was a high relative correspondence between the two ratings involved: one referring to the constituent and the other to the conjunction. The correspondence as expressed in the $z$ differences was relative, in the same way as a correlation makes abstraction of level and variance.

The extensional proximity measure (based on the membership ratings) and the intensional proximity measure (based on the feature-importance ratings) were analyzed using a split-plot ANOVA (Kirk, 1982), with the constituent factor and the syntactic position factor as withingroup (or repeated measures) factors and the three concepts as a between-group factor, and with the $\mathbf{4 0}$ exemplars (or, for the intensional data, the 30 features) as units of observation. In the analysis of the membership ratings, a significant effect of the dominance factor was found $\left[F(1,117)=63.34, p<.0001\right.$, effect size $\left.\omega^{2}=.184\right]$, the dominant constituent being closer to the conjunction $(0.35$ vs. 0.85$)$. Also syntactic position yielded a significant difference $\left[F(1,117)=36.6, p<.0001, \omega^{2}=\right.$ $.011]$ : The constituent functioning as the relative clause modifier was closest to the conjunction (mean proximity of 0.54 , relative to 0.66 for the head noun constituent). The interaction of the dominance factor and the syntactic position factor was significant $[F(1,117)=6.23, p<$ $.014]$, but with a very small effect size $\left(\omega^{2}=.001\right)$; the same was true for the three-way interaction of the dominance factor, the syntactic position factor, and the concept factor $\left[F(2,117)=3.30, p<.04, \omega^{2}=.001\right]$. Despite this very small effect size, it is interesting to look at the three-way interaction. Figure 1 displays all cell proximity means at the three levels of the concept factor. The figure shows a clear presence of the dominance effect in the three conjunctions and of the syntactic position effect in the sports/games data and the machines/vehicles data. The interaction of the two asymmetry phenomena was very different for pets/birds (Figure 1a), sports/games (Figure 1b), and machines/vehicles (Figure 1c).

In the analysis of the intensional proximity measures, a significant effect was found for the dominance factor $\left[F(1,87)=8.78, p<.004\right.$, effect size $\left.\omega^{2}=.034\right]$, be-

Table 3

Correlations Between Constituents and Conjunctions

\begin{tabular}{|c|c|c|c|c|c|c|}
\hline Description & birds & pets & sports & games & vehicles & machines \\
\hline \multicolumn{7}{|c|}{ Membership Ratings } \\
\hline Concept as the head noun & .765 & .257 & .915 & .589 & .811 & .287 \\
\hline Concept in the relative clause & .776 & .207 & .978 & .701 & .972 & .481 \\
\hline \multicolumn{7}{|c|}{ Feature-Importance Ratings } \\
\hline $\begin{array}{l}\text { Concept as the head noun } \\
\text { Concept in the relative clause }\end{array}$ & $\begin{array}{l}.415 \\
.640\end{array}$ & $\begin{array}{l}.254 \\
.396\end{array}$ & $\begin{array}{l}.878 \\
.746\end{array}$ & $\begin{array}{l}.665 \\
.584\end{array}$ & $\begin{array}{l}.192 \\
.761\end{array}$ & $\begin{array}{l}-.065 \\
-.105\end{array}$ \\
\hline
\end{tabular}


cause the importance ratings of the features for the dominant concept were more strongly related to the conjunction than were the corresponding ratings for the nondominant constituent. Neither a main effect of syntactic position nor an interaction between syntactic position and dominance was found. However, the syntactic position $\times$ concept interaction was significant $[F(2,87)=6.43, p<.0025$, $\left.\omega^{2}=.008\right]$. This appears to have been due to the fact that the noncommutativity effect occurred for pets and birds and for machines and vehicles, whereas the reverse effect appeared for the correlations for sports and games.

Because the former ANOVAs might be criticized for the a priori selection of the dominant concept for each conjunction (which was nevertheless based on independent resemblance ratings), and because the (weak) threeway interaction for the membership ratings jeopardizes interpretations of the main effects and of the dominance $x$ syntactic position interaction effect, the proximity measures for each conjunction were analyzed separately in a randomized block factorial ANOVA design (Kirk, 1982) with two double-leveled repeated measures factors: syntactic position and constituent. The results of these separate analyses confirmed the conclusions that were derived from the correlational analyses and from Figure 1. In the analysis of the membership ratings for the pets and birds data, a significant effect for the constituent factor was found $\left[F(1,117)=46.91, p<.0001, \omega^{2}=.173\right]$ : the membership ratings for the bird constituent resembled the ratings for the conjunction better than did the ratings for pets. For the sports and games data and the machines and vehicles conjunction, significant effects were found for syntactic order $\left[F(1,117)=9.21, p<.003, \omega^{2}=.029\right.$; and $F(1,117)=9.13, p<.0031, \omega^{2}=.029$, respectively] and for the constituent factor $[F(1,117)=51.32$, $p<.0001, \omega^{2}=.176$; and $F(1,117)=68.14, p<$ $.0001, \omega^{2}=.240$, respectively]. As in the correlation data reported previously, sports and vehicles could be identified as the dominant constituents.

In the separate analyses of the feature-importance ratings for each conjunction, no significant effects were found for the pets and birds data. For the two other conjunctions, only the dominance effect reached significance $\left[F(1,87)=6.08, p<.0156, \omega^{2}=.031\right.$, for the sports and games data; $F(1,87)=8.22, p<.0052, \omega^{2}=.038$, for the machines and vehicles data]. Again, sports and vehicles were found to be closer to the conjunction.

Analysis of covariance. Next, an attempt was made to control statistically for the possible intensional information in the extensional proximities and for the extensional information in the intensional proximities. For this purpose, covariates were constructed. First, an intensionally based measure for the membership ratings of each exemplar was constructed as a weighted sum of the feature applicabilities for that exemplar, the weights being the feature-importance values. (In other words, the exemplar $\times$ feature applicability matrix was postmultiplied with the feature-importance ratings for the four category types.) Like the direct membership ratings, this measure was also $z$-transformed, and absolute $z$ differences were
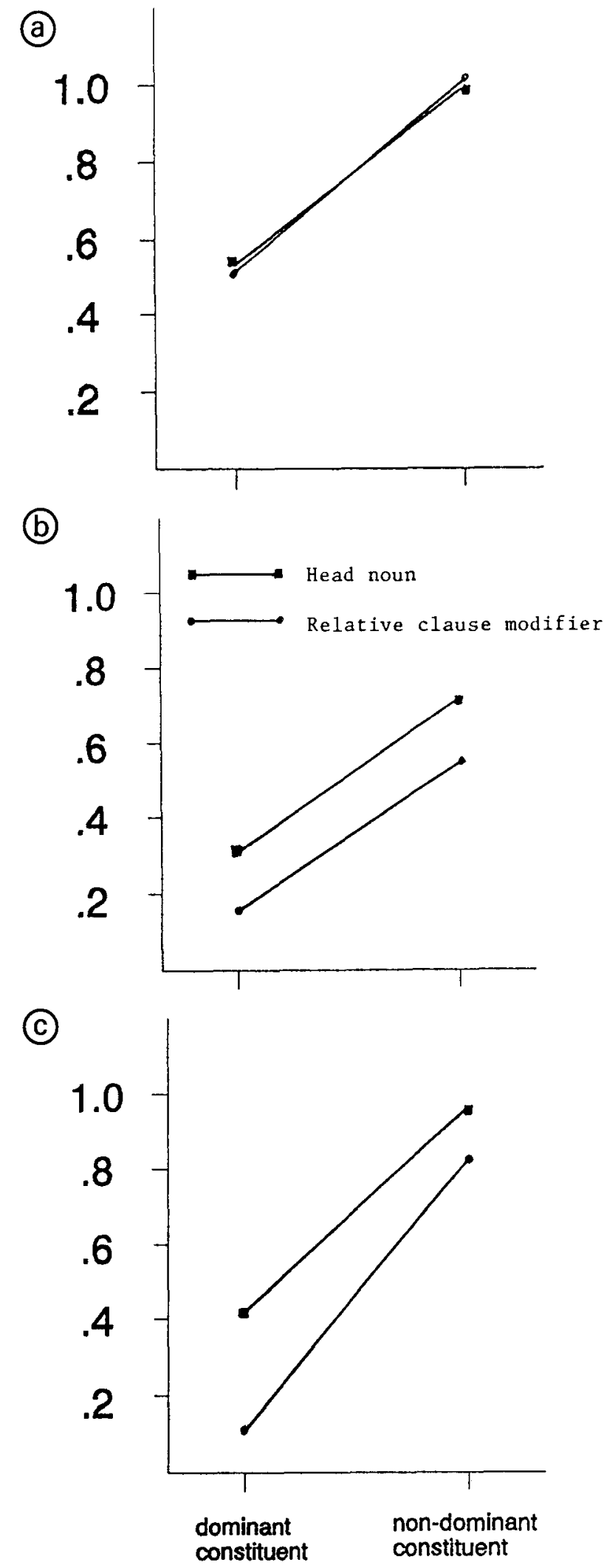

Figure 1. Interactions of the dominance and syntactic position factor in the overall ANOVA of the category membership ratings, depicted separately for (a) birds/pets, (b) sports/games, and (c) vehicles/machines. 
then derived between the measures for the constituents and the measures for the two versions of the conjunctions. Similarly, an extensionally based measure for the importance ratings of each feature was constructed as a weighted sum of the exemplars to which this feature applied, the weights being the membership rating values. (In other words, the exemplar $\times$ feature applicability matrix was premultiplied with the membership ratings for the four category types.) Like the direct feature-importance ratings, this measure was also $z$-transformed, and absolute $z$ differences were then derived between the constituents and the two versions of the conjunction. As a result, intensional and extensional covariates were available for the analysis of extensional proximities ( $z$-transformed exemplar memberships) and intensional proximities ( $z$-transformed feature-importance ratings), respectively. These covariates allowed us to control for an intensional basis in the analysis of the extensional data (membership ratings) and to control for an extensional basis in the analysis of the intensional data (feature-importance ratings).

An overall ANCOVA of the extensional proximity data with the intensionally based covariates of the three conjunctions together yielded a significant effect for the dominance factor $[F(1,116)=16.79, p<.0001]$. The dominant constituent was closer to the conjunction than was the nondominant constituent (.34 vs. .83$)$. The syntactic position factor was also significant $[F(1,116)=34.63$, $p<.0001$, with mean proximities of .65 and .53 for the concept as the head noun and for the concept as the relative clause modifier, respectively. Finally, the interaction of the concept factor and the syntactic position factor was significant $[F(1,116)=4.88, p<.029]$, indicating that the syntactic position effect was stronger for a dominant constituent than for a nondominant constituent. An overall ANCOVA of the intensional proximities with the extensionally based covariates did not yield significant effects for the three conjunctions, the concept factor, the syntactic position, or the interactions.

ANCOVAs for the extensional proximity data of the three conjunctions separately yielded a significant constituent effect in all three analyses $[F(1,116)=5.96, p<$ .0162 , for the pets and birds data; $F(1,116)=63.25$, $p<.0001$, for the sports and games data; $F(1,116)=$ $19.27, p<.0001$, for the vehicles and machines data]. The noncommutativity effect reached significance for the sports and games data and for the vehicles and machines data $[F(1,116)=8.84, p<.0036$, and $F(1,116)=$ $4.10, p<.0451$, respectively], but was not significant for the pets and birds data. Similar ANCOVAs on the intensional proximities (with the extensionally based covariates) yielded no significant effect for any of the conjunctive concepts, the dominance factor, or the syntactic position factor.

\section{Discussion}

We discuss first the question of dominance and noncommutativity effects in extensional and intensional data, based mainly on the results of the ANOVAs, and then the issue of whether the two asymmetry effects have an extensional or intensional basis.

The results suggest that, when people describe conjunctions of semantic concepts as relative clauses, one of the two constituents often dominates the other. This dominance effect showed up when the subjects were asked for extensional information (rating the category membership of a set of exemplars) and when they were asked for intensional information (rating the importance of a set of features for defining a category). The ANOVAs on the three conjunctive concepts together, the separate ANOVAs, and the correlations between the membership ratings for the constituents and for the conjunctions all provide strong support for an extensional dominance effect. In the conjunctions birds, sports, and vehicles consistently dominate pets, games, and machines, respectively. Also, an intensional dominance effect was observed (i.e., a dominance effect in the feature-importance ratings). Only in the separate ANOVA on the pets and birds data, where birds was still the dominant constituent, the difference did not reach significance. In sum, these results, together with the results of Hampton $(1987,1988)$, indicate that the dominance effect is a stable phenomenon at the intensional and extensional levels.

The picture seems more complicated for the noncommutativity effect. Our results indicate that syntactic position had an effect: The influence of a constituent on the conjunction was larger when the constituent was in the relative clause than when it functioned as the head noun. However, this effect does not seem as stable as the dominance effect. The effect appeared for the sports and games data and for the machines and vehicles data, but was reversed for pets and birds. There was a slight tendency for the extensional noncommutativity effect to be stronger for the dominant concepts than for the nondominant concepts. These extensional results are in line with the findings of Hampton (1988) and Chater et al. (1990), but the effect size $\left(\omega^{2}\right)$ clearly indicates that the effect, when it appears, is rather small. Concerning the intension, a nonsignificant noncommutativity effect was observed for all three conjunctions. Hence, the noncommutativity effect on intension seems even weaker than the similar effect on extension.

Hampton (1988) suggested that the locus of both asymmetry effects lies in the intension. Within the framework of his composite prototype model, he explained the dominance effect by assuming that the intension for the conjunction is composed of a mixture of attributes from both constituents, but with more attributes from the dominant concept (Hampton, 1988, p. 26). Similarly, Hampton explained the noncommutativity effect by proposing "that attributes from the relative clause concept are given greater importance in the conjunction and have a greater chance of being inherited by the composite prototype" (Hampton, 1988, p. 26). Hampton found indirect evidence for both explanations in his 1987 study on featureimportance ratings.

The results of our ANCOVAs can serve as a clue for answering the question of whether both asymmetry ef- 
fects have mainly an intensional or an extensional basis. When intensional information was statistically controlled, the dominance effect in membership ratings remained significant in all three conjunctions and the noncommutativity effect still characterized data for sports and games and for vehicles and machines. When extensional information was statistically controlled, the dominance and noncommutativity effects for the feature-importance ratings disappeared.

The effectiveness of the statistical control for extensional and intensional information from the featureimportance and membership ratings, respectively, depends on the reliability of these ratings and on the quality of the exemplar $\times$ feature applicability matrix. Reliabilities above .90 for all three exemplar $\times$ feature applicability matrices were obtained, and both the membership ratings and the feature-importance ratings had a high reliability, with the exception of the feature-importance ratings for the vehicles/machines data. However, the reliability of the membership ratings was superior to the reliability of the feature-importance ratings. This difference can be explained by the inclusion of nonmembers as exemplars in this experiment, while no features were included that were applicable either to any of the constituents or to the conjunction. As a result, the set of features was more homogenous, which may have caused the difference in reliability. The difference in reliability of the membership feature-importance ratings somewhat blurs the results of the ANCOVAs. In the ANCOVA of the extensional data, the dependent variable was more reliable than the covariate, whereas the opposite was true in the ANCOVA of the intensional data. This might explain the finding that the asymmetry effects disappeared in the ANCOVA of the intensional data and did not disappear in the analyses of the extensional data. Yet, the differences in reliability of the membership feature-importance ratings were considerable only for the vehicles/machines data, whereas they were almost negligible for the pets/birds and sports/games data. Nevertheless, the asymmetry effects disappeared in the ANCOVA of the data from all three conjunctions. It is therefore unlikely that differences in reliability account for the ANCOVA results, which may be considered evidence for an extensional basis of the dominance and noncommutativity effects.

A number of considerations concerning the nature of the ratings, the small number of conjunctions, and the choice of the concept pairs used in Experiment 1 led us to conduct a second experiment to further investigate the stability of the two asymmetry effects, as well as the source of these effects.

\section{EXPERIMENT 2}

Experiment 2 was conducted to remedy four weaknesses of Experiment 1. First, the number of concept conjunctions used in Experiment 1 was rather small. Additional data on more conjunctions, therefore, seemed advisable to investigate the stability of the two asymmetry effects. Second, the three conjunctions used were taken from the conjunctions studied by Hampton $(1987,1988)$ and Chater et al. (1990). An investigation of the presence and source of the asymmetry effects in other (new) concept conjunctions would be desirable. Third, as explained above, the reliability of the feature-importance ratings was generally lower than the reliability of the membership ratings. In particular, the low reliability of the featureimportance ratings for the vehicle/machine conjunction was somewhat troublesome. New data, with uniformly high reliabilities, would strengthen our argument. Fourth, in Experiment 1 feature importance was measured on a scale that varied between very important and clearly unrelated. Such a scale does not differentiate between features that are unrelated in the sense of being orthogonal to category membership (e.g., whether or not a creature has an owner is independent of its being a bird) and features that are actually false-that is, negatively related to category membership (e.g., having fur is false of birds). This ambiguity in the scale could have rendered the covariate based on intensions less apt. A scale where subjects are asked for some measure of cue validity (e.g., "Does possession or absence of the feature-indicate category membership, and, if so, how strongly?') would not be subject to this criticism. Otherwise, the ambiguity in the scale could also be (partly) responsible for the lower reliability of the intensional ratings.

\section{Method}

Subjects. The subjects were 60 first-year students from the departments of Linguistics and Theology, 30 first-year students of communication management, and 37 research assistants from the Psychology Department of Leuven University. All subjects were volunteers. Each subject participated in (1) a feature- and exemplar-generation task, (2) two rating tasks (category membership and featureimportance ratings), (3) a dominance judgment task, or (4) an exemplar $\times$ feature judgment task.

Material. Two new conjunctive concepts were used. The first one was the conjunction of office equipment and writing implements, and the second the conjunction of shoes and sports equipment.

Procedure. As the procedure was almost identical to that of Experiment 1 , it will be described briefly, with an emphasis on the differences. Again, 40 subjects participated in the two rating tasks; the order of the two tasks was counterbalanced. Category membership was rated for two lists of 40 exemplars, consisting of 10 exemplars of each of the two constituents, 10 exemplars of the conjunction, and 10 exemplars of a rest category. The subjects were asked to indicate membership ratings on a 7-point scale, ranging from very typical to not typical at all. For the feature-importance rating task, the subjects rated two lists of 40 features: 10 features taken from a feature-generation task for each of the two constituents, 10 features for the conjunction, and 10 features of the rest category. ${ }^{3}$ In the instructions, the subjects were asked to rate whether or not the features were relevant for indicating membership and, if so, how strongly. Ratings were again made on a 7-point scale, ranging from -3 to +3 . For each list of 40 exemplars and each list of 40 features, 10 different subjects were asked to make the ratings for each of four category types (two constituents and the conjunction described in two ways). ${ }^{4}$

For the two pairs of constituents, an a priori criterion of which constituent was dominant was obtained by asking 47 subjects ( 30 students of communication management and 17 research assistants) to complete a resemblance rating task similar to that used in Ex- 
Table 4

Estimated Reliability Coefficients of the Ratings

\begin{tabular}{lcc}
\hline & \multicolumn{2}{c}{ Ratings } \\
\cline { 2 - 3 } writing implements & Membership & Feature Importance \\
office equipment & .972 & .912 \\
writing implements that are also office equipment & .945 & .916 \\
office equipment that are also writing implements & .966 & .935 \\
shoes & .949 & .891 \\
sports equipment & .992 & .883 \\
shoes that are also sports equipment & .987 & .841 \\
sports equipment that are also shoes & .965 & .875 \\
\hline
\end{tabular}

periment 1 . They indicated how much both constituents resembled the conjunction on a 10-point rating scale, ranging from 1 (not at all) to 10 (very much).

Finally, a group of 20 students was asked to judge the applicability of the features to the exemplars. Ten subjects completed the 40 features $\times 40$ exemplars matrices for each of the two conjunctions, and their 1/0 answers were summed, as in Experiment 1.

\section{Results}

Reliability. The reliabilities of the membership and feature-importance ratings, estimated by applying the Spearman-Brown formula to the split-half correlation (as in Experiment 1), are given in Table 4. Most of the coefficients were above .90 , with all membership ratings being only slightly more reliable than the corresponding featureimportance ratings. Reliability estimates for the exemplar $\times$ feature applicability judgments were .93 and .91 for the office equipment/writing implements and shoes/sports equipment conjunctions, respectively. These values are comparable to the estimates for the conjunctions used in Experiment 1.

Identification of the dominant constituents. The mean resemblance ratings of both constituent concepts and their corresponding conjunctions are shown in Table 5. Shoes resembled the shoes/sports equipment conjunction significantly more than did sports equipment $[F(1,46)=7.77$, $p<.01]$. Writing implements resembled the writing implements/office equipment conjunction more than did office equipment $[F(1,46)=21.24, p<.0001]$. These results allowed us to identify shoes and writing implements as the dominant constituents in their corresponding conjunctions.

Correlations and analysis of variance. Both asymmetry effects were investigated using correlation methods and ANOVAs. Correlations between the membership

Table 5

Mean Resemblance Ratings for Constituents and Conjunctions

$\begin{array}{ll}\text { writing implements } & 8.702 \\ \text { office equipment } & 6.553 \\ \text { shoes } & 7.659 \\ \text { sports equipment } & 6.191\end{array}$

ratings for the constituents and the two different relative clause descriptions of the conjunction are given in Table 6 . The correlations for the dominant constituents (shoes and writing implements) were always higher than the corresponding correlations for the nondominant constituents. Correlations for the feature-importance ratings are also shown in Table 6. The dominance effect was present in both descriptions of the office equipment/writing implements conjunction, but a reverse effect showed up in the shoes/sports equipment conjunction. The noncommutativity effect was reflected in the correlations for the membership ratings and, to a small extent, in the correlations of the feature-importance ratings of the shoes/sports equipment conjunction, but was absent in the feature-importance rating correlations for the office equipment/writing implements data.

Measures of proximity between the constituents and their respective conjunctions were calculated as described in Experiment 1 and were again subjected to ANOVAs. A split-plot ANOVA on the membership ratings resulted in a significant dominance effect with a large effect size $\left[F(1,78)=59.28, p<.0001, \omega^{2}=.714\right]$. The dominant constituents were clearly closer to the conjunction than were the nondominant constituents (with mean proximities of .36 and .85 , respectively). Also, the noncommutativity effect on the membership ratings was significant $\left[F(1,78)=6.32, p<.05, \omega^{2}=.002\right]$. The constituent functioning as the relative clause modifier was closest to the conjunction (mean proximity of 0.58 , relative to 0.63 for the head noun constituent), but the small effect size indicated that this noncommutativity effect was of minor importance. The analysis of the feature-importance ratings revealed no main effects for dominance or noncommutativity, although the dominance $\times$ concept interaction did reach significance $[F(1,78)=6.31, p<.025]$. The effect size was also rather small $\left(\omega^{2}=.025\right)$. This interaction showed up because a minor dominance effect was detected for the writing implements/office equipment data. The mean proximity measures were .33 and .42 , respectively; a separate ANOVA revealed this difference to be significant $\left[F(1,78)=163.5, p<.0001, \omega^{2}=.398\right]$. The opposite effect was found for the shoes/sports equip- 
Table 6

\begin{tabular}{|c|c|c|c|c|}
\hline Description & $\begin{array}{c}\text { writing } \\
\text { implements }\end{array}$ & $\begin{array}{c}\text { office } \\
\text { equipment }\end{array}$ & shoes & $\begin{array}{c}\text { sports } \\
\text { equipment }\end{array}$ \\
\hline \multicolumn{5}{|c|}{ Membership Ratings } \\
\hline Concept as the head noun & .943 & .488 & .795 & .387 \\
\hline Concept in the relative clause & .956 & .575 & .856 & 442 \\
\hline \multicolumn{5}{|c|}{ Feature-Importance Ratings } \\
\hline Concept as the head noun & .912 & .856 & .646 & .820 \\
\hline Concept in the relative clause & .910 & .858 & .682 & .839 \\
\hline
\end{tabular}

ment data. The mean proximity measures were .61 and .41 , respectively $\left[F(1,78)=37.03, p<.0001, \omega^{2}=\right.$ .1431 . The effect for the shoes/sports equipment data on feature-importance ratings disagreed with the effect on membership ratings and, most importantly, with the dominance relation of shoes implied by the resemblance ratings.

Analyses of covariance. Because the dominance effect and the noncommutativity effect occurred in the extensional data but not in the intensional data, no ANCOVA was needed. However, because a separate analysis for each of the two conjunctions yielded different results for these effects, we investigated covariation for each conjunction separately. No commutativity effect, but a reversed dominance effect with a minor effect size, emerged for the intensional data of the shoes/sports equipment conjunction. Hence, further analysis did not seem warranted for these data. The noncommutativity effect was not significant for the writing implements/office equipment data, but a regular dominance effect was found in the extensional and intensional data, although the latter effect was only marginally significant. Therefore, covariates were constructed to control statistically for the possible intensional information in the extensional proximities and for the extensional information in the intensional proximities. An ANCOVA showed that the dominance effect remained very significant for the extensional proximity data $[F(1,116)=92.91, p<.0001]$ and remained marginally significant for the intensional proximity data $[F(1,116)=$ $3.98, p<.0485]$.

\section{Discussion}

The results of Experiment 2 were generally consistent with the results of Experiment 1. Again, the dominance effect was present in the membership ratings for the two new conjunctions. For the feature-importance ratings, the dominance main effect was not significant, although the expected effect was found for one conjunction. For this one particular conjunction, the intensional dominance effect did not disappear in the ANCOVA, but the effect size indicated that this intensional effect was much smaller than the comparable dominance effect for the corresponding extensional data. Finally, the noncommutativity effect, which was much weaker than the dominance effect, was in line with the findings in Experiment 1 and with the findings of Hampton $(1987,1988)$. Furthermore, the noncommutativity effect only occurred in the membership ratings. However, the small effect size-and the fact that it dis- appeared in the separate analyses in each concept-strengthened our conjecture that the noncommutativity effect was not a very strong phenomenon.

The findings of Experiment 2 indicate that the dominance effect was a robust phenomenon. It showed up in almost all conjunctive concepts studied by Hampton (1987, 1988) and Chater et al. (1990), as well as in the two new conjunctions used in Experiment 2. The noncommutativity effect, however, seems very weak in terms of the variance in the data that it explains. The results of Experiment 2 also suggest that our concern about the lower reliability of the feature-importance ratings (compared with the membership ratings), as well as about the ambiguity of the meaning of the feature-importance ratings in Experiment 1, was not of crucial importance for the phenomena studied here, because the results of both experiments clearly favored the extensional nature of the dominance effect, and the same seems true for the noncommutativity effect, whenever it shows up.

\section{GENERAL DISCUSSION}

In the two experiments described in this paper, we replicated the dominance effect in concept conjunctions that was described by Hampton $(1987,1988)$ for membership and feature-importance ratings. The noncommutativity effect turned out to be a much weaker, almost negligible effect in terms of the amount of variance it explains. In all cases and in both experiments, the intensional effects disappeared in the ANCOVA, did not appear at all, or was smaller than the extensional effects.

Hampton's (1988) findings (and our findings, which are essentially the same) do not necessarily contradict our interpretation of the locus of both asymmetry phenomena, because it is possible that it is through mediation of exemplars that the conjunction inherits more features, as well as more important features from the dominant constituent and from the relative clause modifier. In this case, the two effects are still to be expected in the featureimportance ratings, but their locus is extensional. In other words, it is possible that subjects, when asked for intensional information (e.g., feature-importance ratings), answer these questions by checking the applicability of features on previously generated exemplars with respect to dominance; this hypothesis implies that features of the best exemplars of a conjunction are more applicable to the dominant constituent than to the nondominant constituent. 
Hence, for the conjunctions used in Experiment 1, we examined how many of the 10 most generated exemplars (in the conjunction) were characterized more by the most typical features of the dominant constituent than by the most typical features of the nondominant constituent in data from a generation study (Storms et al., 1992). We found that 7,9, and 10 exemplars (for birds/pets, sports/ games, and vehicles/machines, respectively) had more features from the dominant constituent than from the other exemplars. If the locus of the dominant effect is extensional, and if the dominance effect in membership ratings is mediated through features, one would expect the best features of the conjunction to characterize better the exemplars of the dominant constituent than the exemplars of the nondominant constituent. In fact, it was found that, out of the 10 most generated features of the conjunctive concept, 5, 5, and 9 (for pets/birds, sports/games, and machines/vehicles, respectively) characterized typical exemplars of the dominant constituent more often than those of the nondominant constituent. It must be emphasized that these findings are based on the generation of features and exemplars and, hence, are an independent source of evidence.

Although the results reported here favor an extensional interpretation, more direct empirical research is needed to bring this question to a definite answer. In the experiments presented here, the evidence is based primarily on statistical methods for controlling how important feature importance (intension) is for the effects on membership ratings and for controlling how important membership (extension) is for the effects on feature-importance ratings. Further research may be set up, for instance, using "thinking-aloud"' studies or using a priming approach in which the effects of feature priming and exemplar priming are compared, for instance, on response times, to identify the underlying process in a more experimental way.

Finally, it must be stressed that the results presented here were obtained using conceptual combinations that are described as relative clauses. Though processes similar to the ones proposed in this article may be present in other forms of conceptual combinations (e.g., in adjective-noun combinations like brown apple, the feature is rotten may be generated through exemplars), the generality of the presented results to noun-noun combinations and other kinds of concept conjunctions needs further investigation.

\section{REFERENCES}

Chater, N., Lyon, K., \& Myers, T. (1990). Why are conjunctive categories overextended? Journal of Experimental Psychology: Learning, Memory, \& Cognition, 16, 497-508.

Clark, E. V., Gelman, S. A., \& Lane, N. M. (1985). Compound nouns and category structure in young children. Child Development, 56, 84-94.

CLARK, H. H. (1983). Making sense of nonce sense. In G. B. Flores d'Arcais \& R. Jarvella (Eds.), The process of understanding language (pp. 297-331). New York: Wiley.

Clark, H. H., \& Marshall, C. R. (1981). Definite reference and mutual knowledge. In A. K. Joshi, B. L. Webber, \& I. A. Sag (Eds.),
Elements of discourse understanding (pp. 10-61). Cambridge: Cambridge University Press.

Downing, P. (1977). On the creation and use of English compound nouns. Language, 53, 810-842.

HAMPTON, J. A. (1987). Inheritance of attributes in natural concept conjunctions. Memory \& Cognition, 15, 55-71.

Hampton, J. A. (1988). Overextension of conjunctive concepts: Evidence for a unitary model of concept typicality and class inclusion. Joumal of Experimental Psychology: Leaming, Memory, \& Cognition, $14,12-32$

Hampton, J. A., \& Dubois, D., (1993). Psychological models of concepts: Introduction. In I. Van Mechelen, J. Hampton, R. S. Michalski, \& P. Theuns (Eds.), Categories and concepts: Theoretical views and inductive data analysis (pp. 11-33). London: Academic Press.

KIRK, R. E. (1982). Experimental design: Procedures for the behavioral sciences (2nd ed.). Belmont, CA: Brooks/Cole.

Kunda, Z., Miller, D. T., \& Claire, T. (1990). Combining social concepts: The role of causal reasoning. Cognitive Science, 14, 551-577.

Medin, D. L., \& SHOBEN, E. J. (1988). Context and structure in conceptual combination. Cognitive Psychology, 20, 158-190.

MuRPHy, G. L. (1988). Comprehending complex concepts. Cognitive Science, 12, 529-562.

ShOBen, E. J. (1991, May). Predicating and nonpredicating conceptual combinations. Paper presented at the meeting of the Midwestern Psychological Association, Chicago, IL.

Smith, E. E., \& Osherson, D. N. (1984). Conceptual combination with prototype concepts. Cognitive Science, 8, 357-361

Smith, E. E., Osherson, D. N., Rips, L. J., \& KeAne, M. (1988). Combining prototypes: A selective modification model. Cognitive Science, 12, 485-527.

SPRINGER, K., \& MURPHY, G, L. (1992). Feature availability in conceptual combination. Psychological Science, 3, 111-117.

Storms, G., Van Mechelen, I., \& De Boeck, P. (1992). Structural analysis of the intension and extension of semantic concepts. Unpublished manuscript.

WISNIEWSKI, E. J., \& GeNTNER, D. (1991). On the combinatorial semantics of noun pairs: Minor and major adjustments. In G. B. Simpson (Ed.), Understanding word and sentence (pp. 241-284). Amsterdam: Elsevier.

\section{NOTES}

1. In the feature-generation study (Storms et al., 1992), subjects were also asked to give features defining the rest categories (e.g., features of animals that are neither pets nor birds). The results indicate that almost no defining features for these rest categories could be generated: Not a single feature, for any of the three rest categories, reached a production frequency larger than two. Because the sample of features was very small and unreliable (due to the low production frequency), we decided to include only the features defining the two constituents and the conjunction.

2. Estimating the reliability of the resemblance ratings caused some problems, because only six different resemblance ratings were asked. In spite of the unreliability of a correlation coefficient based on only six pairs of data, the Spearman-Brown formula was applied to the splithalf correlation, because we could think of no better alternative. A coefficient of .84 was found.

3. Notice that the number of features in the rating task was increased from 30 to 40 . Whereas the feature lists in Experiment 1 did not include 10 features of the rest category (contrary to the exemplar lists that contained 40 exemplars), we decided to treat features and exemplars as similarly as possible in Experiment 2 by adding 10 features of the rest category to the 10 features of each constituent, as well as the 10 features of the conjunction.

4. In defining the rating scales as described here, both scales tap a single continuum in Dutch-that is, the ends of the two scales are defined by polar opposites. Membership ratings vary from very typical to not typical at all, and feature-importance ratings vary from very important (for indicating category membership) to not important at all. 
APPENDIX A

Exemplars for the Sports/Games Conjunction in Dutch (and the English Translation)

\begin{tabular}{llll} 
vier-op-een-rij & (four-in-a-row) & wielrennen & (cycling) \\
hockey & (hockey) & slapen & (sleeping) \\
mens-erger-je-niet & (ludo) & touwtje-springen & (rope-jump) \\
voetbal & (football) & turnen & (gymnastics) \\
rugby & (rugby) & dieren verzorgen & (holding pets) \\
op cafe gaan & (going to a pub) & ganzenspel & (game of gooze) \\
volleybal & (volleyball) & dansen & (dancing) \\
schaken & (chess) & luieren & (laze away the day) \\
haasje-over & (leap frog) & praten & (chatting) \\
tennis & (tennis) & korfbal & (korfball) \\
petanque & (a French ball game) & zwemmen & (swimming) \\
handbal & (handball) & muziek beluisteren & (music listening) \\
badminton & (badminton) & kogelstoten & (shot putting) \\
pingpong & (pingpong) & lezen & (reading) \\
monopolie & (Monopoly) & verspringen & (long jump) \\
paardrijden & (horse riding) & tikkertje & (tag) \\
dammen & (checkers) & verstoppertie & (hide and seek) \\
winkelen & (shoping) & basketbal & (basketball) \\
biljart & (billiards) & basebal & (baseball) \\
lopen & (running) & TV kijken & (watching TV) \\
\hline
\end{tabular}

APPENDIX B

Examples of Features for the Sports/Games Conjunction in Dutch (and the English Translation)

\begin{tabular}{ll}
\hline eist een scheidsrechter & (requires a referee) \\
vals spelen kan & (possible to cheat) \\
kan een beroep zijn & (can be a profession) \\
is prestatiegericht & (achievement oriented) \\
kan binnen gebeuren & (can be done inside) \\
is doel-op-zich & (is a goal on its own) \\
vergt attributen & (requires attributes) \\
gebeurt in groep & (done in group) \\
is aangenaam & (is pleasant) \\
heeft geen nut & (has no use) \\
gebeurt op een terrein & (is done on a field) \\
is vermoeiend & (is tiring) \\
eist bepaalde kledij & (requires special clothes) \\
kan binnen en buiten & (is possible indoors and outdoors) \\
is competitief & (is competitive) \\
gebeurt in water & (done in the water) \\
is gevaarlijk & (is dangerous) \\
kan ook allen & (can be done alone) \\
natuurlijke bezigheid van kinderen & (natural activity of children) \\
gespeeld met een bal & (played with a ball) \\
kan ook buiten & (can be done outside) \\
is goed voor de conditie & (is good for one's condition) \\
is aktief & (is active) \\
eist training & (requires training) \\
in ploegen & (in teams) \\
vergt verstandelijk aktiviteit & (requires mental activity) \\
heeft regels & (has rules) \\
is gezond & (is healthy) \\
vrijwillige deelname & (voluntary participation) \\
is ontspannend & (is relaxing) \\
\hline &
\end{tabular}

